\title{
RNFL variation in gestational diabetes mellitus: An optical coherence tomography based study
}

\author{
Minu Sasikumar, ${ }^{1, *}$, C. V. Anthrayose (Andrews) Kakknatt ${ }^{2}$, Monsy T Mathai ${ }^{3}$ \\ ${ }^{1}$ Junior Resident, ${ }^{2}$ Professor and HOD, ${ }^{3}$ Professor, Dept. of Ophthalmology, Jubilee Mission Medical College and Research \\ Institute, Thrissur, Kerala, India
}

*Corresponding Author:

Email: minu2688@gmail.com

\begin{abstract}
Purpose: To study RNFL thickness in gestational diabetes mellitus based on OCT measurements.

Methods: Case-control study with

Inclusion criteria: 2groups of age 18-40yrs at 32-34weeks gestation

Group 1: Healthy pregnant women

Group 2: Pregnant women with GDM

Exclusion Criteria: Preexisting hypertension/retinal diseases/renal disease/vascular disease/glaucoma/IOP $>21 \mathrm{mmHg} /$ cataract/ refractive error correction more than or equal to +/-5D (spherical) or +/-3D (cylinder).

Grouping-Study Design: more than 2 unrelated group study.

Sample Size: $n=182$

Procedure: After detailed history taking \&.written consent detailed ophthalmic examination is done Patients are classified into group1\&2. Using CIRRUS HD OCT peripapillary RNFL is measured in superior, inferior, nasal and temporal fields.

Results: Peripapillary RNFL showed significant thinning in GDM patients when compared to healthy pregnant female

Conclusion: Peripapillary RNFL thinning in GDM patients measured in their third trimester suggest a neurodegenerative process preceding microvascular changes in gestational diabetes mellitus. The degree of retinal thinning at the level of macula correlated with the glycemic status of the patients. Thereby making it mandatory to carry out routine evaluation of retina with the help of OCT in all GDM patients to detect early neurodegenerative changes and to keep the blood glucose levels in normal range to prevent the occurrence of diabetic retinopathy.
\end{abstract}

Keywords: Gestational diabetes mellitus, RNFL thinning, Neurodegeneration.

\section{Introduction}

Numerous physiological changes occur in pregnancy-increase in ocular blood flow being one of them. Development of diabetes during pregnancy cause an added stress to this hyper perfusion. Gestational diabetes mellitus carries a high risk of development of type 2 diabetes later in life together with maternal and child morbidity. Thus retinopathy screening of gestational diabetes mellitus becomes crucial.

Purpose: To study peripapillary RNFL thickness in gestational diabetes mellitus based on SD-OCT measurements.

\section{Materials and Methods}

A prospective observational study was carried out on the patients attending the Obstetrics and Gynaecology OPD at Jubilee mission medical college. The patients were selected based on the following:

Inclusion Criteria: The study is based on assessment of 2 groups:

Group 1 consists of healthy pregnant women in the age group of 18 to $40 \mathrm{yrs}$ at 32 to 34 weeks of gestation

Group 2 consists of pregnant women with gestational diabetes mellitus (GDM) at 32 to 34 weeks of gestation in the age group 18 to $40 \mathrm{yrs}$.

Diagnostic criteria for GDM (American Diabetes Association 2015 guidelines)-2 step strategy: 50g glucose challenge test (non fasting) with blood sugar measurement after 1 hour: if blood sugar levels more than or equal to $140 \mathrm{mg} / \mathrm{dl}$ proceed to $100 \mathrm{~g}$ Oral Glucose Tolerance Test (OGTT). GDM is diagnosed when 2 or more blood sugar values meet or exceed: fasting: $95 \mathrm{mg} / \mathrm{dl} ; 1$ hour:180mg/dl;2 hour: $155 \mathrm{mg} / \mathrm{dl} ; 3$ hour: $140 \mathrm{mg} / \mathrm{dl}$.

Exclusion Criteria: The exclusion criteria for both groups are as follows:

Pregestational diabetes mellitus, pre-existing hypertension, retinal and macular diseases, renal disease, vascular disease, arteritis, cataract, refractive error correction more than or equal to +/-5D (spherical) or +/-3D (cylinder). Use of drugs like hydroxychloroquine, oral contraceptives and antipsychiatric medications.

Sample Size: Based on the study performed by Gokhan Acmaz et al; "Assessment of macular peripapillary nerve fibre layer and choroidal thickness changes in pregnant women with gestational diabetes mellitus, healthy pregnant women and healthy non pregnant women”; Medical Sci Monit. 2015;21;1759-1764 sample size was calculated based on changes in foveal central thickness in healthy pregnant and in gestational diabetes mellitus ${ }^{12}$

$$
\begin{aligned}
& \mathrm{n}=182 \text { using the formula } \\
& \mathrm{n}=(\mathrm{S} 1+\mathrm{S} 2)^{2}(\mathrm{Z} 1-\mathrm{a} / 2+\mathrm{Z} 1-\hat{\mathrm{a}})^{2} \\
& (\mathrm{X} 1-\mathrm{X} 2)^{2}
\end{aligned}
$$


S1-SD of group 1(healthy pregnant)

S2-SD group 2 (GDM)

$\mathrm{X} 1-\mathrm{Mean}$ group 1 (healthy pregnant)

$\mathrm{X} 2-\mathrm{Mean}$ group $2(\mathrm{GDM})$

$\mathrm{X} 1=224.59$

$\mathrm{X} 2=213.84$

$\mathrm{S} 1=30.1$

$\mathrm{S} 2=16.82$

$\mathrm{n}=91 \times 2=182$

$\mathrm{Z} 1-\mathrm{a} / 2=1.96$

$\mathrm{Z} 1-\mathrm{a}=80 \%$

Mean and standard deviation shall be calculated in each of the 9 quadrants in macula and 4 quadrants of peripapillary region. These values will be compared between the 2 groups -healthy pregnancy and GDM.

Procedure: A detailed history was taken about any previous or existing ophthalmic diseases or surgeries, co existing medical and surgical conditions. After obtaining written consent from patient, detailed ophthalmic examination was done including refraction, oblique illumination, assessment of anterior segment, intraocular pressure measurement and direct ophthalmoscopy. Patients are then classified into group 1 and 2. Group 1 includes healthy pregnant females at 32 weeks of gestation in the age group 18 to $40 \mathrm{yrs}$ and Group 2 includes patients with gestational diabetes mellitus at 32 weeks of gestation in the age group 18 to 40yrs. Based on ADA criteria, GCT \& OGTT are done in group 2 patients.

Optical coherence tomography using CIRRUS HD OCT without pupillary dilatation and under the same intensity of dim room lighting -by the same specialist was done in both groups \& following parameter was evaluated:

Peripapillary RNFL thickness measured in, superior, inferior, nasal and temporal outer fields.

Statistical Analysis: The data obtained was coded and entered in microsoft excel sheet and analysed using Statistical software package for social sciences (SPSSvs22). Chi square test was used to find the $p$ value. $\mathrm{p}$ value $<0.05$ is considered statistically significant.

Statistical tests used were mean, standard deviation, student t test, Chi square test, Karl Pearson Coefficient

\section{Results}

Data was collected from 182 patients and patients meeting the inclusion criteria was categorised into 94 healthy pregnant group and 88 pregnant women with gestational diabetes mellitus. Data obtained from 364 eyes was coded and entered in Microsoft excel sheet and analyzed using SPSS vs 22.

Age: The table shows age distribution of the patients. As the $p$ value is not significant age is not a comparable factor between healthy pregnant and GDM patients. Thereby age does not influence retinal thickness at the level of RNFL in both groups. $79 \%$ of the healthy pregnant and $65 \%$ if GDM patients belonged to the 21 30 age group thereby forming the majority.

Table 1:

\begin{tabular}{|l|c|c|c|c|}
\hline \multirow{2}{*}{ Group } & \multicolumn{3}{|c|}{ Age group } & \multirow{2}{*}{ p-value } \\
\cline { 2 - 4 } & $11-20$ & $21-30$ & $31-40$ & \\
\hline Control & $8(8.5 \%)$ & $74(78.7 \%)$ & $12(12.8 \%)$ & \multirow{2}{*}{0.06} \\
\hline GDM & $12(13.6 \%)$ & $55(62.5 \%)$ & $21(23.9 \%)$ & \\
\hline Total & $20(11 \%)$ & $129(70.9 \%)$ & $33(18.1 \%)$ & \\
\hline
\end{tabular}

Peripappilary RNFL Thickness: 364 eyes were subjected to peripapillary RNFL assessment using OCT. Peripapillary RNFL was assessed in 4 quadrants -Superior, inferior, nasal and temporal quadrants together with mean thickness.

Table 2: RNFL thickness in case and controls

\begin{tabular}{|l|c|c|c|c|c|}
\hline \multirow{2}{*}{ RNFL Quadrants } & \multicolumn{2}{|c|}{ Control } & \multicolumn{2}{c|}{ GDM } & \\
\cline { 2 - 6 } & N & Mean \pm SD & n & Mean \pm SD & p-value \\
\hline Peripapillary RNFL Mean & 188 & $100.75 \pm 41.55$ & 176 & $91.16 \pm 13.25$ & 0.004 \\
\hline Peripapillary RNFL Superior & 188 & $127.81 \pm 41.62$ & 176 & $117.27 \pm 19.90$ & 0.003 \\
\hline Peripapillary RNFL Inferior & 188 & $131.41 \pm 59.05$ & 176 & $118.85 \pm 18.24$ & 0.008 \\
\hline Peripapillary Nasal & 188 & $77.46 \pm 49.02$ & 176 & $66.37 \pm 15.10$ & 0.004 \\
\hline Peripapillary Temporal & 182 & $65.57 \pm 25.84$ & 176 & $61.90 \pm 19.72$ & 0.132 \\
\hline
\end{tabular}

All 4 quadrants of peripapillary RNFL analysed showed thinning with significant thinning in all quadrants except temporal and inferior quadrant showing maximum thickness and temporal quadrant showing minimum thickness in both healthy pregnant 
and GDM patients. Maximum thickness recorded was $131.41 \pm 59.05 \mu \mathrm{m}$ in healthy pregnant and

$118.85 \pm 18.24 \mu \mathrm{m}$ in GDM patients.

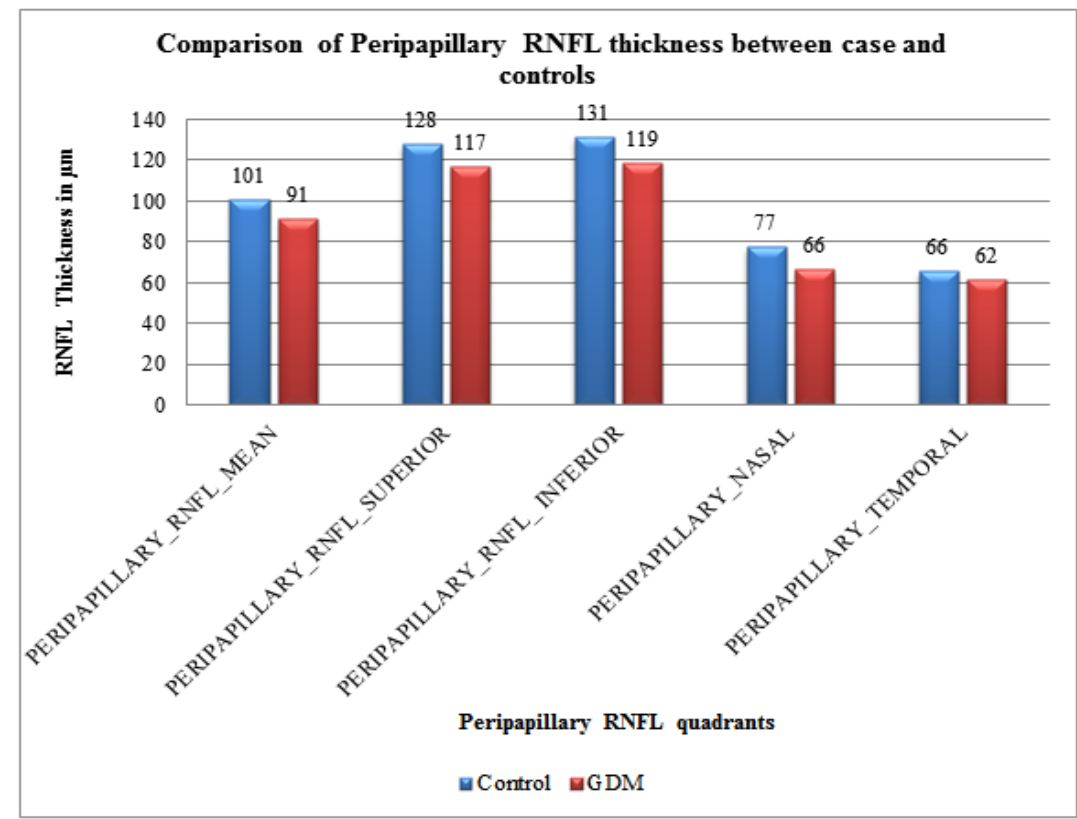

Fig. 2: Comparison of peripapillary RNFL thickness between case and controls

Fasting GTT values showed significant positive correlation with mean RNFL thickness together with superior and temporal RNFL quadrants.1 hr GTT values showed positive correlation with levels of thinning in mean peripapillary RNFL and temporal RNFL thickness thereby reflecting the influence of blood sugar levels on retinal thinning in macula as well as RNFL.

Table 3: Correlation of peripapillary RNFL thickness with glucose tolerance test among GDM patients

\begin{tabular}{|c|c|c|c|c|c|}
\hline & & GDM & GDM & GDM & GDM \\
\hline \multirow[t]{3}{*}{ Peripapillary RNFL Mean } & Pearson Correlation & -0.219 & -0.276 & -0.15 & 0.16 \\
\hline & p-value & 0.044 & 0.011 & 0.17 & 0.147 \\
\hline & $\mathrm{N}$ & 176 & 176 & 176 & 176 \\
\hline \multirow[t]{3}{*}{ Peripapillary RNFL Superior } & Pearson Correlation & -0.219 & -0.198 & -0.094 & 0.141 \\
\hline & p-value & 0.045 & 0.069 & 0.391 & 0.202 \\
\hline & $\mathrm{N}$ & 176 & 176 & 176 & 176 \\
\hline \multirow[t]{3}{*}{ Peripapillary RNFL Inferior } & Pearson Correlation & -0.17 & -0.18 & -0.142 & -0.218 \\
\hline & p-value & 0.12 & 0.1 & 0.194 & 0.047 \\
\hline & $\mathrm{N}$ & 176 & 176 & 176 & 176 \\
\hline \multirow{3}{*}{ Peripapillary RNFL Nasal } & Pearson Correlation & 0.102 & -0.025 & -0.006 & 0.482 \\
\hline & p-value & 0.353 & 0.824 & 0.959 & 0 \\
\hline & $\mathrm{N}$ & 176 & 176 & 176 & 176 \\
\hline \multirow[t]{3}{*}{$\begin{array}{l}\text { Peripapillary RNFL } \\
\text { Temporal }\end{array}$} & Pearson Correlation & -0.322 & -0.332 & -0.155 & -0.085 \\
\hline & p-value & 0.003 & 0.002 & 0.157 & 0.443 \\
\hline & $\mathrm{N}$ & 176 & 176 & 176 & 176 \\
\hline
\end{tabular}

\section{Discussion}

Optical coherence tomography is a medical imaging technique using light to capture three dimensional images from scattering area. Long wavelength light penetrates into scattering media. It is useful in images requiring micrometre resolution and millimetre penetration. The principle of optical coherence tomography is low coherence interferometry. It consists of interferometer with low coherence and broad wavelength light source. Light is split into reference arm and recombined from sample arm.

Spectral domain or fourier domain optical coherence tomography extracts spectral data by distributing different optical frequencies into a detected stripe via a 
dispersive element. Thus full depth scan can be acquired with single exposure ${ }^{1,2}$

Numerous physiological changes occur in the body during pregnancy. This includes an increase in cardiac blood flow and volume. A major part of this is increase in ocular blood flow which is diverted to the choroid which in turn is important for maintaining retinal function, intraocular pressure, ocular thermoregulation and uveoscleral aqueous drainage. ${ }^{3-5}$

Development of diabetes during pregnancy carries the risk of developing Type II DM and contributes to both maternal and child morbidity. Placenta secretes hormones like corticotrophin releasing hormone, placental lactogen and progesterone which makes pregnancy a diabetogenic state. ${ }^{101}$ Diabetic retinopathy screening is important due to the fact that patients remain asymptomatic till they develop feature of macular oedema or proliferative diabetic retinopathy. ${ }^{71}$ Confocal scanning laser polarimetry, optical coherence tomography and scanning laser polarimetry are the three methods employed for study of retinal nerve fibre layer thickness of which this study uses optical coherence tomography to assess retinal nerve fibre layer thickness.

There are studies on variations in macular thickness in normal pregnancy and retinal nerve fibre layer thickness in gestational diabetes mellitus. Current study is based on both retinal nerve fibre layer thickness and macular thickness in normal pregnancy and gestational diabetes mellitus.

This study was a prospective observational study conducted 364 eyes of 182 patients with 94 belonging to healthy pregnant and 88 belonging to gestational diabetes mellitus group. It was conducted on all patients attending the Obstetrics and Gynecology Department at Jubilee Mission Medical College for a period of 18 months.

All the patients in the study were assessed at 32 weeks of gestation comparison of Age: Patients were aged between 18 to 40 years and statistical analysis showed no significant correlation of age between 2 groups. Studies by Pradhan et al showed positive correlation of age with foveal thickness and negative correlation of other quadrants of macula with age. ${ }^{6}$ Eriksson et $\mathrm{al}^{7}$ proved foveal thickness decreased with age whereas studies by Chan $\mathrm{A}$ et $\mathrm{al}^{8}$ and Kanai $\mathrm{k}$ et $\mathrm{al}^{9}$ disproved this. In our study on comparing age $p$ value is not significant thereby it does not influence the RNFL variations in either groups. Our study does not show any significant correlation between thinning of various quadrants and age. In our study there was no significant correlation of age with retinal thinning in macula or peripapillary RNFL. Average age was 28 years with maximum subjects in the age group of 21-30 years. $79 \%$ of healthy pregnant and $65 \%$ of GDM patients belonged to this category. Youngest subject was 18 years old and oldest 38 years.
Peripapillary retinal nerve fiber layer thickness: With the help of OCT we have measured RNFL thickness in the peripapillary region by dividing it into 4 quadrants and the mean RNFL thickness. This data was compared between healthy pregnant and GDM patients with all the 4 quadrants showing thinning and with significant thinning in all 3 quadrants -superior, nasal and temporal quadrants. Average RNFL thickness also showed a significant decrease.

This data was compared to normative data presented by Mansoori et al. ${ }^{10}$

Table 4: Comparison of RNFL thickness between
our study and Mansoori et al
\begin{tabular}{|l|c|c|}
\hline Quadrants & Present Study & Mansoori et al \\
\hline $\begin{array}{l}\text { Average } \\
\text { RNFL }\end{array}$ & $100.75 \pm 41.55$ & $113.9 \pm 10.7$ \\
\hline Superior & $127.81 \pm 41.62$ & $138 \pm 18.1$ \\
\hline Inferior & $131.41 \pm 59.05$ & $142.7 \pm 17.4$ \\
\hline Nasal & $77.46 \pm 49.02$ & $104.1 \pm 15.2$ \\
\hline Temporal & $65.57 \pm 25.84$ & $70.6 \pm 10.5$ \\
\hline
\end{tabular}

When comparing values obtained in this study with study done by Mansoori et al in normal subjects the values obtained were lesser in healthy pregnant with GDM patients showing further lower values.

Thickest quadrant was inferior followed by superior, nasal and temporal which was comparable in both studies. No studies have so far recorded the peripapillary RNFL thickness in GDM patients and hence a comparison in this regard was not done.

Studies by Hille W. van Dijk ${ }^{11}$ have shown neurodegenerative changes to occur before vascular changes in TYPE 1 Diabetes Mellitus caused by ganglion cell layer thinning.GDM is also a condition of insulin resistance wherein the same hypothesis can recur thereby explaining the thinning of RNFL. None of the patients showed any diabetic change. Hence it may be hypothetised that the retinal thinning may be precursor for diabetic vascular change. All the GDM patients considered for the study were on insulin therapy.

Similar study on retinal neurodegeneration prior to vasculopathy was also done by Sedat Arikan et al. ${ }^{12} \mathrm{~A} \mathrm{~J}$ Barber et al proved apoptosis of retinal ganglion cells during degeneration in diabetes. ${ }^{13}$ Kanan $\mathrm{Y}$ et al studied the pathway for this neurodegeneration by concluding on the impaired retinal signalling pathway or deficiency of intermediary components for this neurodegeneration and vasculopathy. ${ }^{14}$ Xuebin Liu et al implicated persistent expression of SCOS-3 in the development of retinal insulin resistance. ${ }^{15}$ This degeneration reflected as impaired contrast sensitivity in study conducted by Doss et al. ${ }^{16}$

Chhablani J et al also concluded the event of retinal neurodegeneration prior to vasculopathy reflected as 
decrease in thickness in RNFL and Ganglion cell layer and the necessity of neuroprotection at this stage. ${ }^{17}$

Qin Yang et al confirmed this hypothesis by performing FFA and OCT in murine model with spontaneous type 2 diabetes which showed obvious thinning. Significant alternations in PERG responses and increased retinal ganglion cells (RGCs) apoptosis was observed in diabetic $\mathrm{db} / \mathrm{db}$ mice for 20 -week period. IBA-1 and F4/80 expression (neuro inflammatory markers) in microglia/macrophages became evident at 24 -week period, thus supporting the PERG findings. ${ }^{18}$

Barber $\mathrm{AJ}$ et al also showed that insulin is a survival factor for retinal neurons by activating the PI 3-kinase/Akt pathway and by reducing caspase-3 activation. ${ }^{19}$ Hence from the present study we may conclude that GDM induces retinal neurodegeneration without any vasculopathy which is in turn is prevented by insulin. Insulin gives neuroprotection thus the thinning of retina is less likely to be due to the effect of insulin.

Comparison of blood glucose levels with retinal thinning: The retinal thinning at macula and peripapillary showed positive correlation with levels of fasting and $1 \mathrm{hr}$ GTT levels. $\mathrm{HbA}_{1} \mathrm{c}$ and GCT values did not show any significant correlation with level of thinning. Till now no study has compared the effects of blood sugar levels and retinal thinning. Gokhan Acmaz et $\mathrm{al}^{20}$ has studied retinal thinning in GDM patients and proved the reversal of choroidal thinning with adequate blood sugar control which projects the fact that not merely the presence of gestational diabetes mellitus but the level of uncontrolled blood sugar levels affects the process of retinal neurodegeneration. Kida et al. examined optic nerve head $(\mathrm{ONH})$ blood circulation during 75-gr OGTT and concluded that $\mathrm{ONH}$ circulation decreased in the abnormal glucose tolerance group, attributed partly to the increased endothelin- $1 .{ }^{21}$

The significant positive correlation of GTT fasting and 1 hour values reflect the need for performing OCT along with glucose tolerance test as this helps in preventing early neurodegenerative changes occurring in RNFL. Thus uncontrolled blood sugar values brings about changes in the retinal microcirculation which causes thinning at the level of peripapillary optic nerve head or vice versa as evidence suggests that neurodegenerative changes occur prior to microvascular changes in diabetes.

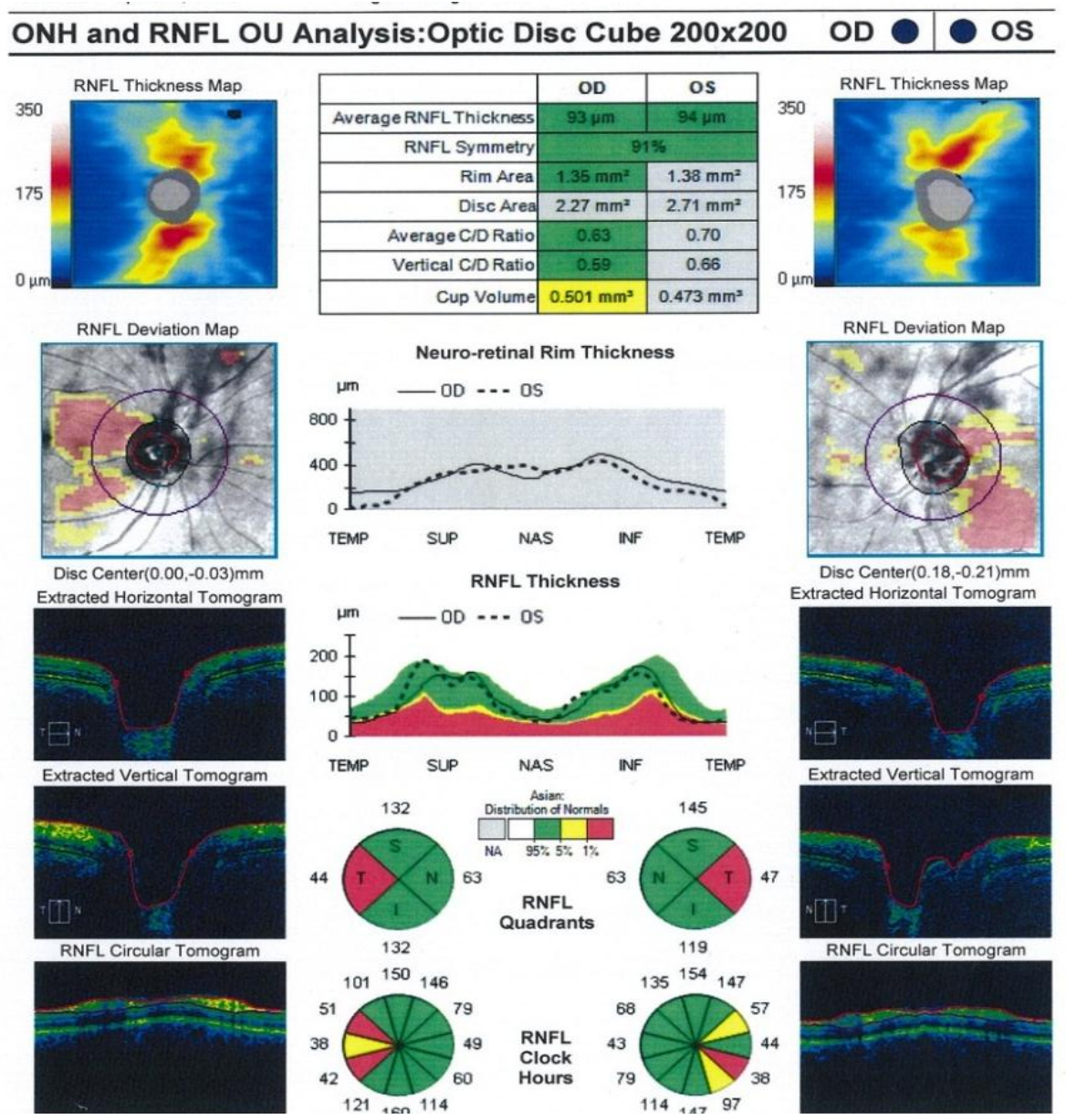




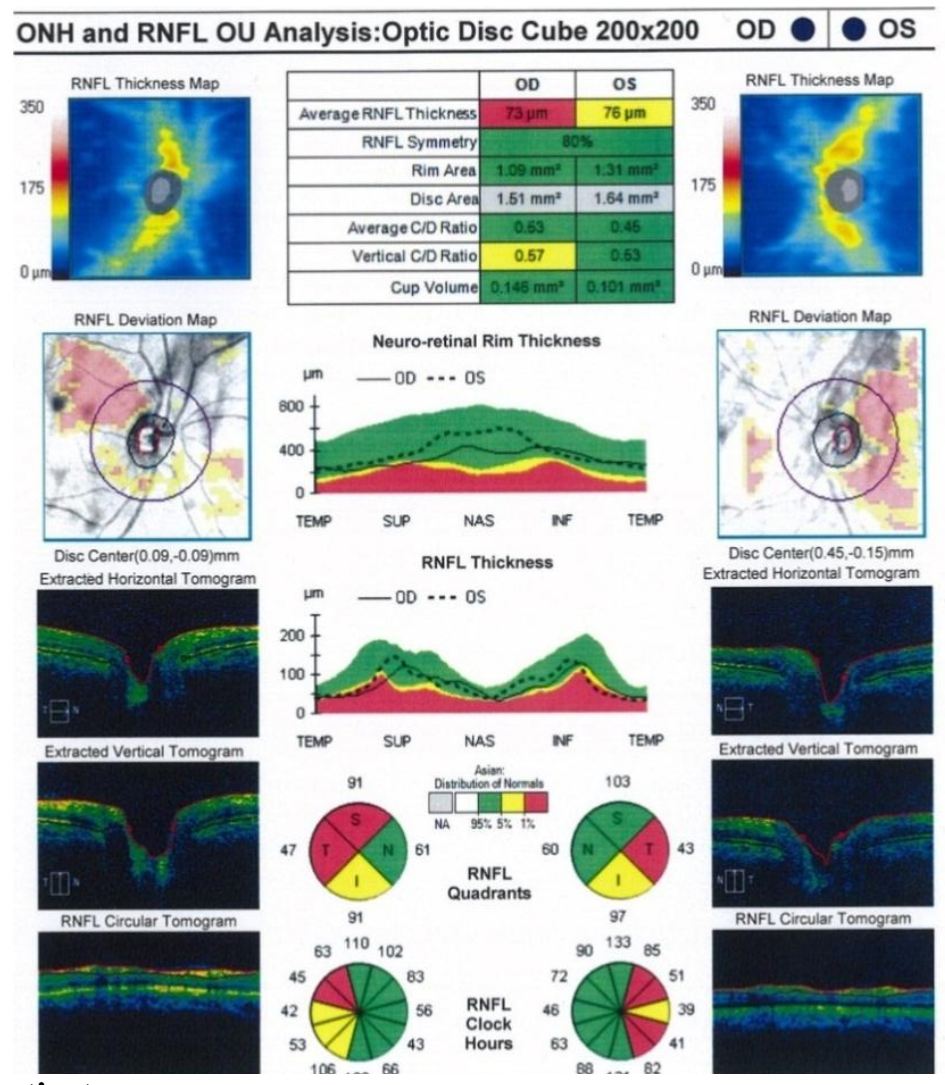

\section{Fig. 3: OCT of GDM patient}

This is a prospective comparative study on the optical coherence tomography changes in normal pregnancy and gestational diabetes mellitus done at Jubilee Mission Medical College \& Research Institute from December 2015 to November 2017. Institutional Ethics Commitee clearance was obtained. The main aim of the study was to analyse variation in thickness in RNFL at the peripapillary region.

The average age distribution of the patients was between 21 to 30 age in both case and controls with majority of the patients being primi gravida. Peripapillary RNFL showed thinning with significant thinning in all quadrants except temporal RNFL

\section{Conclusion}

The detailed analysis of our study which was conducted on 182 patients which included 94 healthy pregnant and 88 GDM patients was compared and reviewed with reported statistics and literature.

Optical coherence tomography performed in these patients at 32 to 34 weeks of gestation revealed thinning of peripapillary RNFL with significant thinning in all quadrants except temporal RNFL. Peripapillary RNFL thinning revealed significant positive correlation with fasting and 1 hour GTT values. Hence it can be concluded that GDM patients with uncontrolled blood sugar was found to develop neurodegeneration in the absence of microvascular changes-a feature observed in insulin dependent diabetes mellitus. Hence it becomes mandatory to perform routine evaluation of retina with the help of OCT along with glucose tolerance test in all GDM patients to detect early neurodegenerative changes and keep blood glucose levels in normal range to prevent the occurrence of diabetic retinopathy.

\section{References}

1. Swanson E.A, Izatt J.A, Hee M.R, Huang D,Lin C.P, Schuman J.S et al ; "In vivo Retinal Imaging by OCT". Opt Lett (1993);vol:18(issue 21);1864-1866.

2. Schmitt, J.M; “Optical Coherence Tomography: A review'. IEEE Journal of Selected Topics in Quantum Electronics (July 1999); vol 5(4):pg 1205-1213.

3. Centofanti M, Miqliardi R, Bonini S, Manni G, Bucci MG, Pesavento CB et al ;"Pulsatile ocular blood flow during pregnancy”. Eur J Ophthalmol;(2002);vol 12(issue 4); pg no:276-280.

4. Chen HC, Newsom RS, Patel V, Cassar J, Mather H, Kohner EM, "Retinal blood flow changes during pregnancy in women with diabetes" Invest ophthalmol vis sci journal(1994); vol 35(issue 8):3199-3208.

5. Nickla DL, Wallman J; "The multifunctional choroid" Prog Retin Eye Res;(2010) ; vol 29(2):144-168

6. Pradhan; Determinants of macular thickness in normal Indian eyes; Journal of clinical ophthalmology; 2013; volume1;issue1;pp. 2320-3897.

7. Eriksson U, Alm A. Macular thickness decreases with age in normal eyes: A study on macular thickness map protocol in Stratus OCT. Br J Ophthalmol 2009;93:144852.

8. Chan A, Duker JS, Ko TH, Fujimoto JG, Schuman JS. Normal macular thickness measurements in healthy eyes 
using Stratus optical coherence tomography. Arch Ophthalmol 2006;124:193-8.

9. Kanai K, Abe T, Murayama K, Yoneya S. Retinal thickness and changes with age. Nippon Ganka Gakkai Zasshi 2002;106:162-5.

10. Mansoori T, Viswanath K, Balakrishna N. Quantification of retinal nerve fiber layer thickness using spectral domain optical coherence tomography in normal Indian population. Indian J Ophthalmol. 2012;60(6):555-8.

11. Hille W. van Dijk, Frank D. Verbraak, Pauline H. B. Kok, Mona K. Garvin, Milan Sonka, Kyungmoo Lee et al; Decreased Retinal Ganglion Cell Layer Thickness in Patients with Type 1 Diabetes IOVS, July 2010, Vol. 51, No 7.

12. 12.Sedat Arikan, Ismail Ersan, Mustafa E rogulu, Mehmet Yılmaz, Hasan Ali Tufan, Baran Gencer et al; Does Retinal Neurodegeneration Seen in Diabetic Patients Begin in the Insulin Resistance Stage; Turk $J$ Ophthalmol. 2016 Dec;46(6):264-269.

13. Barber AJ, Lieth E, Khin S A, Antonetti DA, Buchanan AG, Gardner TW; Neural apoptosis in the retina during experimental and human diabetes- Early onset and effect of insulin. Journal of Clinical Investigation; 1998;783791.

14. Kanan Y, Matsumoto H, Song H, Sokolov M, Anderson $\mathrm{RE}$, Rajala RV;Serine/threonine kinase akt activation regulates the activity of retinal serine/threonine phosphatases; J Neurochem. 2010;113:477-488.

15. Liu X, Mameza MG, Lee YS, Eseonu CI, Yu CR, Kang Derwent JJ et al; Suppressors of cytokine-signaling proteins induce insulin resistance in the retina and promote survival of retinal cells. Diabetes. 2008;57:1651-1658.

16. Dosso AA, Yenice-Ustun F, Sommerhalder J, Golay A, Morel Y, Leuenberger PM; Contrast sensitivity in obese dyslipidemic patients with insulin resistance; Arch Ophthalmol. 1998;116:1316-1320.

17. Chhablani J, Sharma A, Goud A, Peguda HK, Rao HL, Begum VU et al; Neurodegeneration in Type 2 Diabetes: Evidence From Spectral-Domain Optical Coherence Tomography; Invest Ophthalmol Vis Sci. 2015 Oct;56(11):6333-8.

18. Qin Yang, Yidan Xu, Ping Xie, Haixia Cheng, Qinglu Song, Tu Su et al; Retinal Neurodegeneration in $\mathrm{db} / \mathrm{db}$ Mice at the Early Period of Diabetes; Journal of Ophthalmology Volume 2015 (2015), Article ID 757412, 9 pages.

19. Barber AJ, Nakamura M, Wolpert EB, Reiter CE, Seigel GM, Antonetti DA et al; Insulin rescues retinal neurons from apoptosis by a phosphatidylinositol 3-kinase/Aktmediated mechanism that reduces the activation of caspase-3. J Biol Chem. 2001;276:32814-32821.

20. Gokhan Acmaz, Mustafa Atas, Ahmet Gulhan, Banu Acmaz, Fatma Atas, Huseyin Aksoy; "Assessment of macular peripapillary nerve fibre layer and choroidal thickness changes in pregnant women with gestational diabetes mellitus, healthy pregnant women and healthy non pregnant women"; Medical Sci Monit. 2015;21;17591764.

21. Kida T, Sugiyama T, Oku H, et al. Plasma endothelin-1 levels depress optic nerve head circulation detected during the glucose tolerance test. Graefes Arch Clin Exp Ophthalmol. 2007;245:1289-93. 